\title{
Can the Genericity Assumption Decrease the Rank of a Matrix?
}

\author{
András Recski1*, Áron Vékássy \\ ${ }^{1}$ Department of Computer Science and Information Theory, Faculty of Electrical Engineering and Informatics, Budapest \\ University of Technology and Economics, H-1521 Budapest, P. O. B. 91, Hungary \\ *Corresponding author, e-mail: recski@cs.bme.hu
}

Received: 11 June 2020, Accepted: 27 December 2020, Published online: 02 February 2021

\begin{abstract}
The genericity assumption, supposing that the nonzero parameters of a system are algebraically independent transcendentals over the field of the rationals, often helps for the mathematical modelling of linear systems. Without this condition nonzero expansion members of a determinant can cancel out each other, decreasing the rank of a matrix. In this note we show that under some circumstances an increase is also possible. This counterintuitive phenomenon is explained using some tools from matroid theory, and is illustrated by a classical network of Carlin and Youla.
\end{abstract}

\section{Keywords}

circuit analysis, linear multiports, genericity

\section{Introduction}

The genericity assumption is often applied in the qualitative study of linear systems. For example, the network of Fig. 1 which contains a current controlled current source $i_{4}=c i_{2}$, is uniquely solvable if and only if $R_{2}+R_{3}+(c+1) \times R_{5}=0$ does not hold. However, such an equation (or any other a priori given algebraic equation) cannot be satisfied if we suppose that the parameters of the network are algebraically independent transcendental numbers.

Similar situations arise in other engineering disciplines as well. For example, let the graph of a planar bar-and-joint framework be isomorphic to the Kuratowski graph $K_{3,3}$. Then the framework will be infinitesimally rigid [1] unless the six joints are on a common conic section [2] this condition is equivalent to a quadratic equation among the 12 coordinates.

Therefore, one expects that special values of some system parameters can only decrease the rank of a matrix (see a more precise formulation in Section 2). However, we show that an increase is also possible. After a theoretical explanation (which requires some tools from matroid
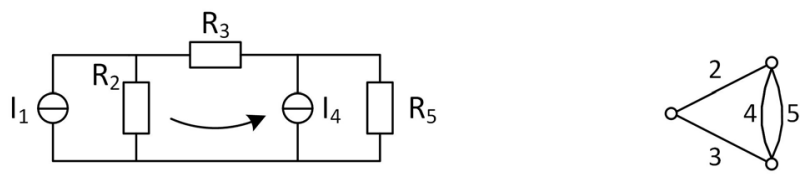

Fig. 1 A network and its graph after replacing the independent current source by an open circuit theory) we illustrate the phenomenon with a classical network of Carlin and Youla [3] and Carlin [4].

For the definitions of the concepts from matroid theory the reader is referred to [1] or [5]. We use standard notations of matroid theory, in particular, / denotes contraction and $\vee$ denotes the union of matroids.

\section{Term rank, genericity and an example}

Let $r(\mathbf{M})$ denote the "usual" rank of a matrix M. A set of nonzero entries in a matrix $\mathbf{M}$ is a transversal if no pair of these entries share the same row or column. The size $t(\mathbf{M})$ of a maximum transversal is the term rank of $\mathbf{M}$. Clearly $t(\mathbf{M}) \geq r(\mathbf{M})$ since the determinant of a square matrix is the signed sum of products of entries along maximum size transversals but these products can cancel out each other. For example, the $p \times q$ matrix with ones in every position has rank 1 while its term $\operatorname{rank}$ is $\min (p, q)$ for every $p, q \geq 1$.

Such cancellations are impossible if the matrix is generic, that is, if its nonzero entries are algebraically independent over the field of the rational numbers. Hence the rank of the matrix can only decrease if we drop the genericity assumption. For example, if the independent current source $I_{1}$ is replaced by an open circuit in the network of Fig. 1, we have a system of 8 equations and the coefficient matrix has rank 8 or it reduces to 7 if $R_{2}+R_{3}+(c+1) \times R_{5}=0$ holds. Similarly, there are plenty of examples that the degree of freedom of a 
network composed of multiports, capacitors and inductors decreases if we drop genericity.

Remark. There are several definitions of genericity in the literature. For a critical comparison the reader is referred to [6].

However, if a multiport arises as the interconnection of some others, a somewhat surprising phenomenon arises: the rank of the final multiport can also increase if we drop genericity.

In order to explain this recall that the interconnection of some multiports $M_{1}, M_{2}, \ldots$ into a single multiport $M$ is described by a graph $G$ as illustrated in Fig. 2 where two 2-ports are interconnected to form a three-port. Both the ports of the original multiports and those of the obtained one correspond to edges of $G$. Observe that if terminal nodes of a multiport have internal connections (like the ones belonging to ports 1 and 2 of the upper 2-port) then this is reflected by $G$. Throughout we shall suppose that $G$ contains neither loops nor bridges (coloops). However, $G$ need not be 2-vertex-connected.

If a multiport $M$ is described by $\mathbf{A u}+\mathbf{B i}=\mathbf{0}$ with $r(\mathbf{A} \mid \mathbf{B})=r$ then the column space matroid of the matrix (A|B) will be denoted by $\mathbb{m}(M)$. Recall that if $\mathbf{C}$ is a nonsingular $r \times r$ matrix and we change the description of the multiport to the equivalent one $(\mathbf{C A} \mid \mathbf{C B})$ then the matroid remains the same.

The edge set $E$ of the interconnection graph $G$ is the union of the set $E_{I n t}$ of the internal edges (corresponding to the ports of the multiports $\left.M_{1}, M_{2}, \ldots\right)$ and the set $E_{E x t}$ of the external edges (corresponding to the ports of the resulting multiport $M$ ). Since each port has a voltage and a current, let $E^{U}$ and $E^{I}$ denote the set of all the voltages and that of all the currents, respectively. These sets can also be decomposed into $E_{\text {Int }}^{U} \cup E_{E x t}^{U}$ and into $E_{\text {Int }}^{I} \cup E_{E x t}^{I}$, respectively.

Let $q$ denote the direct sum of the cycle matroid of $G$ on the set $E^{I}$ and the cocycle matroid of $G$ on the set $E^{U}$. Let $A^{\prime}$ denote the direct sum of the matroids $m\left(M_{1}\right), m\left(M_{2}\right), \ldots$ on the set $E_{I n t}$. Let us extend $\mathcal{A}^{\prime}$ with loops on the set $E_{E x t}$ to

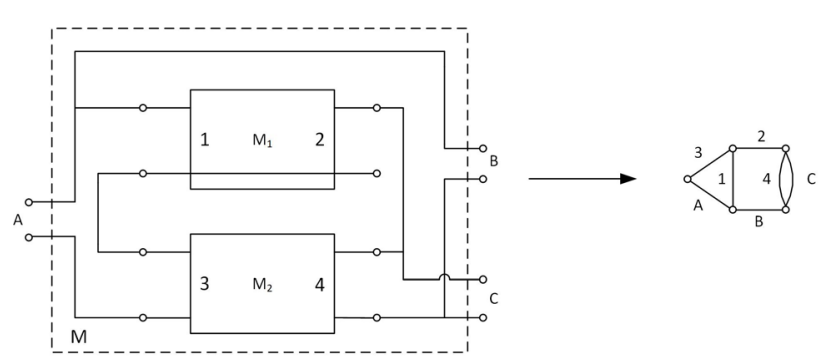

Fig. 2 Two 2-ports $M_{1}$ and $M_{2}$ interconnected to form a 3-port $M$, and the graph $G$ of the interconnection obtain a matroid $\mathcal{A}$. Observe that both matroids $\mathcal{G}$ and $\mathcal{A}$ are defined on $E^{U} \cup E^{I}$. If the genericity assumption holds then $m(M)=(\mathfrak{g} \vee \mathcal{A}) /\left(E_{\text {Int }}^{U} \cup E_{\text {Int }}^{I}\right)$ see [7, 8].

Suppose now that we drop the genericity and a cancellation occurs. Usually it decreases the rank of the multiport, but if this cancellation happens to be within the set $\left(E_{I n t}^{U} \cup E_{I n t}^{I}\right)$, then one will contract a subset of smaller rank, hence the rank of the final multiport can also increase.

A classical example of Carlin and Youla [3] (Fig. 3) is revisited to illustrate this.

The matroid $m(C)$ of a 3-port circulator $C$ is isomorphic to the cycle matroid of a complete graph on 4 vertices, see $[6,7,8]$. Hence the matroids $\boldsymbol{q}$ and $\boldsymbol{A}$ are the cycle matroids of the graphs of Figs. 4(a) and (b), respectively, their union is the uniform matroid $\boldsymbol{u}_{12,11}$, that is, a circuit of length 12 . The subgraph formed by the edges with subscripts less than 6 is circuit-free and after contracting them we obtain a length 2 circuit, corresponding to a resistor, as expected in the generic case.

If we put $R_{1}=1, R_{2}=-1$, the number of linearly independent equations decreases from 11 to 10 . The coefficient

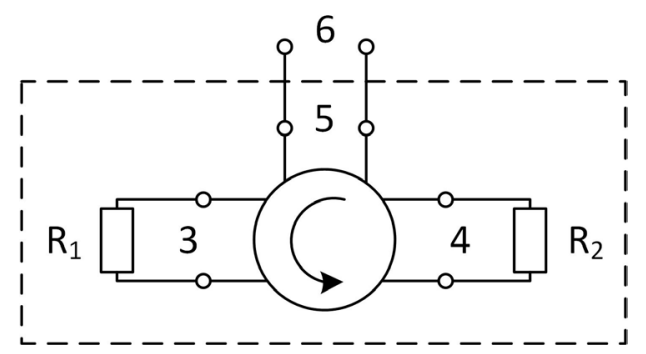

Fig. 3 The circulator network of Carlin and Youla [3]

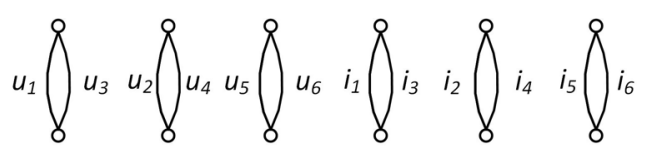

a)
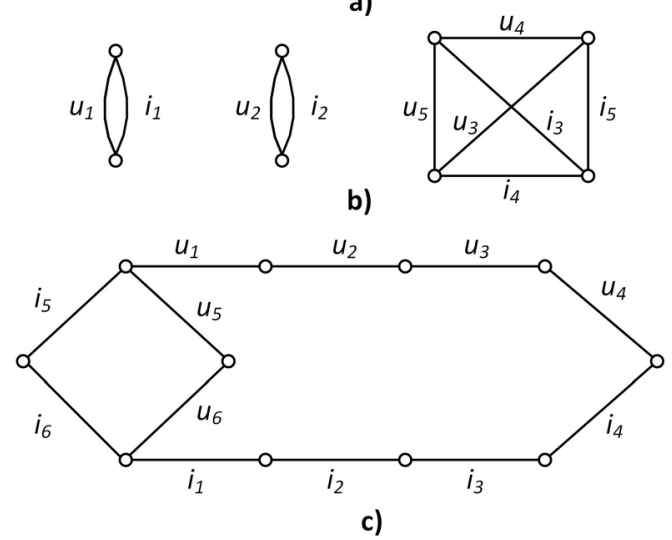

Fig. 4 The graphs representing the matroids in the example; a); b); c) 
matrix of the system of equations determines a matroid which is different from $\mathfrak{G \vee A}$ but happens to be graphic again, see the graph of Fig. 4(c) for its representation - observe that it is not the length 12 circuit anymore but a graph containing two linearly independent circuits. The subgraph formed by the edges with subscripts less than 6 is still circuit-free, its contraction leads to two loops, corresponding to the norator - a decrease of the final rank, as expected.

If we put $R_{1}=-1, R_{2}=1$, the number of linearly independent equations remains 11 , but the matroid of the system becomes the direct sum of a length 8 circuit and 4 bridges, corresponding to $u_{5}, u_{6}, i_{5}$ and $i_{6}$. Hence the subgraph formed by the edges with subscripts less than 6 does contain a circuit and after contracting these edges we obtain two bridges, corresponding to the nullator. Since the rank of the contracted subgraph decreased, we experience an increase in the rank of the resulting 1-port.

\section{Further discussion}

For the complete understanding of the behavior of this network it might be instructive to add some further remarks:

1. If $R_{1}+R_{2}=0$ still holds but $R_{1} R_{2} \neq-1$ then the number of linearly independent equations remains 11, but the matroid of the system becomes the direct sum of a length 10 circuit and 2 bridges, corresponding to $i_{5}$ and $i_{6}$. Hence we obtain an open circuit.

2. If $R_{1}+R_{2} \neq 0$ but $R_{1} R_{2}=-1$ still holds then the number of linearly independent equations remains 11 , but the matroid of the system becomes the direct sum of a length 10 circuit and 2 bridges, corresponding to $u_{5}$ and $u_{6}$. Hence we obtain a short circuit.

3. If none of the relations $R_{1}+R_{2}=0$ and $R_{1} R_{2}=-1$ holds then the number of linearly independent equations remains 11 , but the matroid of the system need not necessarily become $\boldsymbol{U}_{12,11}$, as expected for the generic case. If, for example, we put $R_{1}=1$ and $R_{2}=1$, the matroid becomes the direct sum of a length 8 circuit and 4 bridges, corresponding to $u_{2}, u_{4}, i_{2}$ and $i_{4}$. Nevertheless, this does not change the final result since we must contract the subgraph formed by the edges with subscripts less than 6 .

\section{References}

[1] Recski, A. "Matroid Theory and its Applications in Electric Network Theory and in Statics", Springer-Verlag, Berlin, Heidelberg, Germany, 1989. https://doi.org/10.1007/978-3-662-22143-3
4. Recall that in the generic case the network behaves like a resistor. In the "traditional" way, without using matroid theory, we can solve the system of the 11 equations describing the network and obtain the relation between the current and the voltage of port 6 (i.e. the resistance of the port) as a function of the values of the resistances terminating ports 3 and 4 .

$u_{6}=\frac{R_{1} R_{2}+1}{R_{1}+R_{2}} i_{6}$

We can plot this resistance value as a surface, to visualize the behavior of this network, see Fig 5. Aside from the generic case it is immediately clear that:

- if $R_{1}+R_{2}=0$, the resistance of this port is infinite and therefore it behaves like an open circuit, and

- if $R_{1} R_{2}=-1$ holds then this resistance becomes zero and we obtain a short circuit.

\section{Acknowledgement}

The research reported in this paper has been supported by the National Research, Development and Innovation Fund (BME NC TKP2020, Thematic Excellence Program and OTKA\#124171) and by the Higher Education Excellence Program of the Ministry of Human Capacities in the frame of the Artificial Intelligence research area of the Budapest University of Technology and Economics (BME FIKP-MI/ $\mathrm{SC}$ ). The referees' remarks are also gratefully acknowledged.

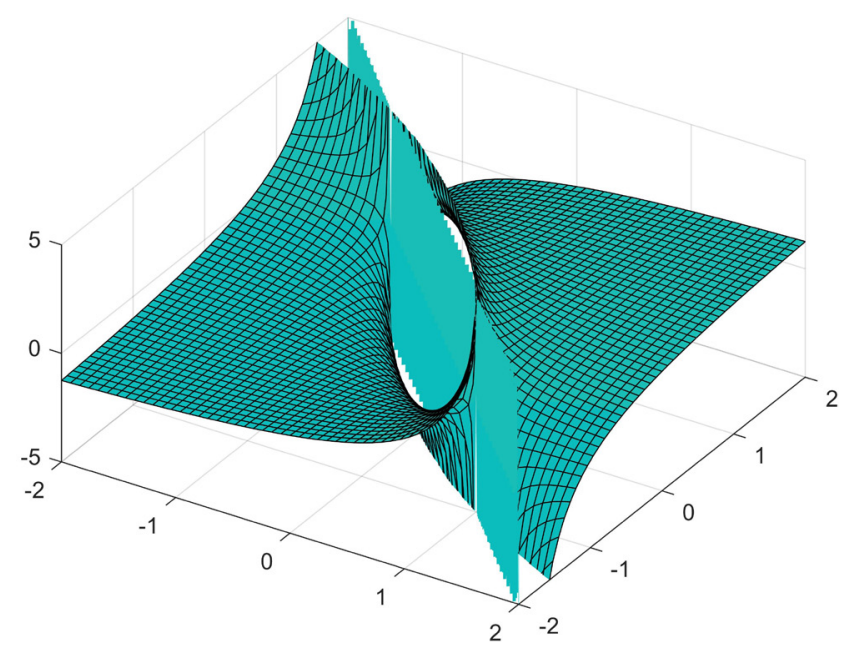

Fig. 5 The resistance of port 6 as a function of $R_{1}$ and $R_{2}$

[2] Bolker, E., Roth, B. G. "When is a bipartite graph a rigid framework?", Pacific Journal of Mathematics, 90(1), pp. 27-44, 1980. https://doi.org/10.2140/pjm.1980.90.27

[3] Carlin, H. J., Youla, D. C. "Network Synthesis with Negative Resistors", Proceedings of the IRE, 49(5), pp. 907-920, 1961. https://doi.org/10.1109/JRPROC.1961.287934 
[4] Carlin, H. "Singular Network Elements", IEEE Transactions on Circuit Theory, 11(1), pp. 67-72, 1964. https://doi.org/10.1109/TCT.1964.1082264

[5] Oxley, J. G. "Matroid theory", Oxford University Press, Oxford, UK, 2006.

[6] Recski, A., Iri, M. "Network theory and transversal matroids", Discrete Applied Mathematics, 2(4), pp. 311-326, 1980. https://doi.org/10.1016/0166-218X(80)90027-X
[7] Recski, A. "Unique solvability and order of complexity of linear networks containing memoryless $n$-ports", International Journal of Circuit Theory and Applications, 7(1), pp. 31-42, 1979. https://doi.org/10.1002/cta.4490070105

[8] Recski, A. "Matroids and Network Synthesis", In: 1980 European Conference of Circuit Theory and Design (ECCTD), Warsaw, Poland, 1980, pp. 192-197. 